\title{
Relación entre el nivel de conocimientos y actitudes sobre la alimentación saludable y el nivel de anemia en gestantes en Tacna
}

\author{
Relationship between the level of knowledge as well as attitudes about \\ healthy eating and the level of anemia in pregnant women in Tacna
}

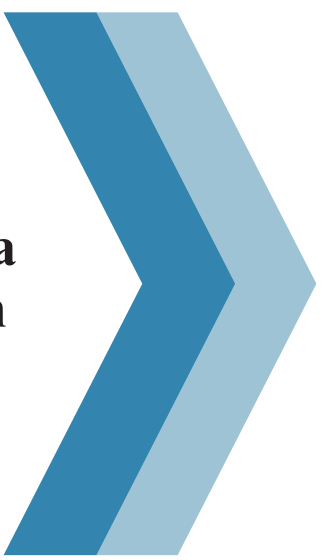

\author{
Edith Rocío Godoy Gonzáles ${ }^{1 a}$ \\ Gladys Gloria Concori Cori ${ }^{\text {b }}$ \\ Leandra Herminia Llanca Ramos ${ }^{1 \mathrm{c}}$ \\ María Yamile Salazar Anco ${ }^{2 \mathrm{~d}}$
}

${ }^{1}$ Escuela Profesional de Obstetricia de la Universidad Nacional Jorge Basadre Grohmann. Tacna, Perú.

${ }^{2}$ Centro de Salud La Natividad, Ministerio de Salud. Tacna, Perú.

${ }^{a}$ Docente, Doctora en Ciencias de la Salud

${ }^{\mathrm{b}}$ Docente, Magister en Tecnología Educativa.

Docente, Doctora en Salud Pública.

Obstetra Asistencial, Magister en Salud Pública.

\section{RESUMEN}

La anemia es una complicación frecuente en el embarazo; por tanto se requiere conocimientos y actitudes sobre alimentación saludable para superarla. Objetivo: Determinar la relación entre el nivel de conocimientos y actitudes sobre alimentación saludable en gestantes anémicas y el nivel de la anemia en la Microred Metropolitana de la Red de Salud Tacna. Material y métodos: Estudio correlacional y de corte transversal en 74 gestantes con anemia, de 18 a 45 años, pertenecientes a la Microred Metropolitana de la Red de Salud Tacna, con reciente dosaje de hemoglobina y consentimiento informado. Los datos se recolectaron en un cuestionario en el consultorio de obstetricia de los cinco establecimientos de salud que conforman la microred, mediante una entrevista a las gestantes. Para el procesamiento y análisis estadístico se utilizó los softwares microsoft excel y SPSS versión 20 para windows. Se presentan tablas y gráficos con pruebas estadísticas Fisher y Chi-cuadrado x2. Resultados: E1 $62,2 \%$ de las gestantes cursaban el tercer trimestre de gestación, el 35,1 \% fueron primigestas, el 44,6\% presentaron sobrepeso, el 33,8\% normopeso y el 6,7 \% delgadez. Se encontró que el 58,1 \% tenía anemia leve y el $41,9 \%$ anemia moderada. El 56,8\% de las gestantes en general presentaron medio o regular nivel de conocimiento sobre alimentación saludable; de esta cantidad, el $31,1 \%$ fue de gestantes con anemia leve y $25,7 \%$ fue de gestantes con anemia moderada. El 67,6 $\%$ de gestantes con anemia presentaron actitud favorable sobre alimentación saludable, de las cuales el 40,6 \% presentaba anemia leve y el $27,0 \%$ presentaba anemia moderada. Conclusiones: Existe relación estadísticamente significativa entre el nivel de conocimiento y actitud sobre alimentación saludable y nivel de la anemia en las gestantes.

Palabras Clave: Alimentación saludable, gestante, anemia, conocimientos, actitudes.

\section{ABSTRACT}

Anemia is a frequent complication in pregnancy; that is why knowledge and attitudes about healthy eating are required to overcome it. Objective: To determine the relationship between the level of knowledge and attitudes about healthy eating in anemic pregnant women and the level of anemia in the Metropolitan Microred of the Tacna Health Network. Material and methods: Correlational and cross-sectional study in 74 pregnant women with anemia, aged 18 to 45 , belonging to the Metropolitan Microred of the Tacna Health Network, with recent dosing of hemoglobin and informed consent. The data were collected in a questionnaire in the obstetrics office of the five health establishments that are part of the micro-network, through an interview with pregnant women. For statistical processing and analysis, it was used the Microsoft Excel and SPSS version 20 software for windows. Tables and graphs are presented with Fisher and Chi-square x 2 statistical tests. Results: $62.2 \%$ of pregnant women were in the third trimester of pregnancy, the $35.1 \%$ were primipara, the $44.6 \%$ were overweight, the $33.8 \%$ were normal weight and the $6.7 \%$ were thin. It was found that the $58.1 \%$ presented mild anemia and the $41.9 \%$ moderate anemia. The $56.8 \%$ of pregnant women in general had a medium or regular level of knowledge about healthy eating; of this amount, the $31.1 \%$ were pregnant women with mild anemia and the $25.7 \%$ were pregnant women with moderate anemia. The $67.6 \%$ of pregnant women with anemia had a favorable attitude on healthy eating, of which the $40.6 \%$ had mild anemia and the $27.0 \%$ had moderate anemia. Conclusions: There is a statistically significant relationship between the level of knowledge and attitude about healthy eating and the level of anemia in pregnant women.

Keywords: Healthy nutrition, pregnant, anemia, knowledge, attitudes. 


\section{Introducción}

Se considera el conocimiento como el primer paso en el proceso de cambio de una conducta porque lo que se sabe o se cree influye en la manera como se actúa (1-3). La actitud es una predisposición aprendida a valorar o comportarse de una manera favorable o desfavorable de una persona, objeto o situación (4).

La alimentación durante el embarazo constituye uno de los aspectos fundamentales a tener en cuenta. Se deben vigilar y recomendar los nutrientes básicos para madre y el desarrollo del feto (5). La anemia afecta principalmente a los niños, las mujeres en edad fértil y las gestantes(6).

La Organización Mundial de la Salud (OMS) establece los rangos de referencia para el diagnóstico de la anemia, según el nivel de hemoglobina en la sangre de las gestantes, el grado de anemia: leve: $\mathrm{Hb}$ 10,0 - 10,9 $\mathrm{g} / \mathrm{dl}$, moderado $\mathrm{Hb} 7,0-9,9 \mathrm{~g} / \mathrm{dl}$, y severo $\mathrm{Hb}<7,0$ $\mathrm{g} / \mathrm{dl}(7)$.

Saidman (8) realizó un estudio para explorar cambios alimentarios, conocimientos sobre alimentación y nutrición, mitos, tabúes y creencias en 117 gestantes sanas mayores de 17 años que concurrieron al control de su embarazo en Buenos Aires Argentina. El autor observó que el $31 \%$ de las embarazadas atribuyó connotaciones negativas a algún alimento; asimismo, se evidenció el desconocimiento sobre la ganancia de peso y sobre la anemia. En el estudio, se resaltó la importancia de diseñar un programa de intervención nutricional, donde los ejes centrales sean el componente educativo y la consejería nutricional priorizando los conocimientos, intereses y creencias identificadas por el grupo de embarazadas.

Guevara (9) realizó una investigación con el objetivo de determinar la relación de conocimientos y actitudes de alimentación saludable con la anemia en gestantes que asistían al programa de psicoprofilaxis Baby Hope de la Clínica Good Hope en Lima. El estudio de enfoque cuantitativo, de diseño no experimental, de corte transversal y de nivel relacional en el que participaron 52 gestantes entre $18-45$ años seleccionadas por conveniencia. El estudio mostró que no existe relación significativa entre el nivel de conocimientos y las actitudes sobre la alimentación saludable y la anemia en las gestantes.

El presente estudio servirá para sistematizar la información, evaluar el nivel de conocimientos y actitudes; asimismo, brindará información sobre el nivel de conocimientos sobre alimentación saludable en gestantes a la Microred Metropolitana de la Red de Salud Tacna. Los resultados permitirán incentivar la implementación de estrategias educativas dirigidas a las gestantes para la promoción de una alimentación saludable y prevención de la anemia en gestantes a través de la consejería con mensajes claves y enfoque intercultural, lo cual contribuirá a mejorar la salud de la madre y el niño por nacer. El objetivo es establecer la relación entre el nivel de conocimientos y la actitud de la gestante con anemia sobre la alimentación saludable, así como la prevalencia de anemia.

\section{Material y métodos}

Este es un estudio correlacional y de corte transversal. Se contó con la participación de 74 gestantes atendidas durante el periodo de noviembre 2018 a abril del 2019, con reciente dosaje de hemoglobina y consentimiento informado. Las gestantes presentaban anemia y fueron atendidas en los centros de salud que conforman la Microred Metropolitana, tales como el C.S. La Natividad, C.S. Bolognesi, el C.S. Leoncio Prado, el C.S. Augusto B. Leguía, y el C. S. Metropolitano.

Para realizar este trabajo se solicitó el permiso a la Dirección de la Red de Salud Tacna y a las gerencias de los establecimientos de salud, la Jefatura del Servicio de Obstetricia y la obstetra responsable de la estrategia sanitaria salud sexual y reproductiva componente materno perinatal de los establecimientos de la Microred.

Se incluyeron gestantes con anemia y que aceptaron voluntariamente participar del estudio. Se excluyeron a las gestantes con problemas de discernimiento o con patologías crónicas, según el registro de gestantes que obra en el servicio de obstetricia de los establecimientos de salud.

La recolección de datos se llevó a cabo mediante el uso de un cuestionario de conocimientos sobre alimentación en la gestación, conformado por 8 preguntas de datos generales y 18 preguntas de opción múltiple, y un test de actitudes alimentarias que utiliza una escala de Likert compuesta de 19 reactivos con opciones de respuesta de 5 alternativas.

El procedimiento de la recolección de datos se realizó después de la consulta, y luego de obtener el resultado de hemoglobina de la primera batería de análisis de la gestante atendida, mediante una entrevista a las gestantes con anemia que acudieron al consultorio de obstetricia, se entrevistó en la sala de espera de los establecimientos de la Microred; dándole tiempo para el llenado de 40 minutos. Los instrumentos de la investigación utilizados para determinar los conocimientos y actitud, se basaron en Guevara (9), los cuales fueron validados para Tacna.

Para el procesamiento y análisis estadístico se utilizó los softwares microsoft excel y SPSS versión 20 para windows. Se presentan tablas y gráficos con 
pruebas estadísticas Fishery Chi-cuadrado $x^{2}$.

En relación a los aspectos éticos, la información obtenida en los cuestionarios a través de las entrevistas fue con consentimiento informado de las gestantes, y se ha mantenido con absoluta confidencialidad.

\section{Resultados}

En la Tabla 1 se muestran las características sociodemográficas de las gestantes atendidas en establecimientos de la Microred Metropolitana de la Red de Salud de Tacna. Así, el 79,7 \% de las gestantes tenía entre 18 a 35 años de edad. Con respecto al estado civil el $77,0 \%$ de las gestantes eran convivientes. El $63,5 \%$ de las gestantes tenía como ocupación ser ama de casa.

Tabla 1. Características sociodemográficas de las gestantes

$\begin{array}{lcc} & \text { Gestantes } \\ \text { Edad } & \mathrm{n} & \% \\ \text { 18 a 25 años } & 29 & 39,2 \\ \text { 26 a 35 años } & 30 & 40,5 \\ \text { 36 a 45 años } & 15 & 20,3 \\ \text { Total } & 74 & 100,0 \\ \text { Estado civil } & & \\ \text { Soltera } & 8 & 10,8 \\ \text { Conviviente } & 57 & 77,0 \\ \text { Casada } & 9 & 12,2 \\ \text { Total } & 74 & 100,0 \\ \text { Ocupación } & & \\ \text { Ejecutivo } & 2 & 2,7 \\ \text { Comerciante } & 12 & 16,2 \\ \text { Ama de casa } & 47 & 63,5 \\ \text { Otros } & 13 & 17,6 \\ \text { Total } & 74 & 100,0\end{array}$

En la Tabla 2 se muestra que de 74 gestantes encuestadas el 100,0 \% de ellas tuvieron gestación única, el $62,2 \%$ de las gestantes señalaron que estaban en el tercer trimestre de gestación, poco más de las tres cuartas partes del total de gestantes tuvo tres o más gestaciones, y el 44,6\% de ellas tenía sobrepeso.
Tabla 2. Características obstétricas de las gestantes atendidas

\begin{tabular}{lcc} 
& \multicolumn{2}{c}{ Gestación actual } \\
Tipo de gestación & $\mathrm{n}$ & $\%$ \\
Único & 74 & 100,0 \\
Total & 74 & 100,0 \\
Trimestre de gestación & & \\
Primer trimestre & 7 & 9,4 \\
Segundo trimestre & 21 & 28,4 \\
Tercer trimestre & 46 & 62,2 \\
Total & 74 & 100,0 \\
$\mathrm{~N}^{\circ}$ de gestaciones & & \\
Primera & 26 & 35,1 \\
Segunda & 23 & 31,1 \\
Tres o más gestaciones & 25 & 33,8 \\
Total & 74 & 100,0 \\
Imc en gestantes & & \\
Delgadez & 5 & 6.7 \\
Normal & 25 & 33.8 \\
Sobrepeso & 33 & 44.6 \\
Obesidad & 11 & 14.9 \\
Total & 74 & 100.0
\end{tabular}

En la Tabla 3, se muestra que de 74 gestantes el 58,1\% tenía un nivel de anemia leve; mientras que el $41,9 \%$ tenía un nivel de anemia moderada.

Tabla 3. Nivel de anemia en gestantes atendidas

\begin{tabular}{lcc}
\hline Nivel de anemia & $\mathrm{n}$ & $\%$ \\
\hline Leve & 43 & 58,1 \\
Moderada & 31 & 41,9 \\
Total & 74 & 100,0
\end{tabular}

En la Tabla 4, se puede apreciar que de 42 gestantes el $56,8 \%$ presentaba medio o regular nivel de conocimiento sobre alimentación saludable; seguido del 23,3\% que consideraba que tenía alto nivel de conocimiento sobre alimentación saludable; mientras que el 20,3\% consideraba que tenía bajo nivel de conocimiento.

Tabla 4. Nivel de conocimientos sobre alimentación saludable en gestantes

\begin{tabular}{|ccc|}
\hline Nivel de conocimientos & $\mathrm{n}$ & $\%$ \\
\hline Bajo & 15 & 20,3 \\
Medio & 42 & 56,8 \\
Alto & 17 & 23,0 \\
Total & 74 & 100,0 \\
\hline
\end{tabular}

En la Tabla 5 se muestra que el $31,1 \%$ de las gestantes con anemia leve tenía un nivel de conocimientos medio 
sobre alimentación saludable; mientras que el $25,7 \%$ de las gestantes con anemia moderada tenía un nivel de conocimiento medio. Por tanto, es posible afirmar que el nivel de conocimiento en alimentación saludable se relaciona significativamente con el nivel de anemia en gestantes.

Tabla 5. Relación entre el nivel de conocimiento y el nivel de anemia en gestantes

\begin{tabular}{|c|c|c|c|c|c|c|c|}
\hline \multirow{3}{*}{$\begin{array}{c}\text { Nivel } \\
\text { de }\end{array}$} & \multicolumn{6}{|c|}{ Nivel de Anemia } & \multirow{3}{*}{ Total } \\
\hline & & \multicolumn{2}{|c|}{ Anemia Leve } & \multicolumn{2}{|c|}{ Anemia Moderada } & & \\
\hline & s & $\mathrm{n}$ & $\%$ & $\mathrm{n}$ & $\%$ & $\mathrm{n}$ & \\
\hline Bajo & & 9 & $12,2 \%$ & 6 & $8,1 \%$ & 15 & $20,3 \%$ \\
\hline Medio & & 23 & $31,1 \%$ & 19 & $25,7 \%$ & 42 & $56,8 \%$ \\
\hline Alto & & 12 & $16,2 \%$ & 5 & $6,8 \%$ & 17 & $23,0 \%$ \\
\hline Total & & 44 & $59,5 \%$ & 30 & $40,5 \%$ & 74 & $100,0 \%$ \\
\hline
\end{tabular}

Prueba exacta de Fisher $=0,037 \quad P$-Valor $=0,000$

En la Tabla 6 podemos observar que de 74 gestantes atendidas, el 67,6\% tenía un nivel de actitud

favorable, además el 32,4\% tenía un nivel de actitud muy favorable.

Tabla 6. Distribución según nivel de actitud en gestantes atendidas

\begin{tabular}{|ccc} 
Nivel de actitud & $\mathrm{n}$ & $\%$ \\
\hline Favorable & 50 & $67,6 \%$ \\
Muy Favorable & 24 & $32,4 \%$ \\
Total & 74 & $100,0 \%$
\end{tabular}

En la Tabla 7 se muestra que el $40,6 \%$ de las gestantes con anemia leve tenían un nivel de actitud favorable sobre alimentación saludable; mientras que solo el $27,0 \%$ de las gestantes con anemia moderada presentaban un nivel de actitud favorable sobre la alimentación saludable.

Tabla 7. Relación entre nivel de actitud y nivel de anemia en gestantes atendidas

\begin{tabular}{lcccccc}
\multicolumn{1}{c}{$\begin{array}{c}\text { Nivel de actitud } \\
\text { en gestantes }\end{array}$} & \multicolumn{2}{c}{ Anemia Leve } & \multicolumn{2}{c}{ Anemia Moderada } & \multicolumn{2}{c}{ Total } \\
& $\mathrm{n}$ & $\%$ & $\mathrm{n}$ & $\%$ & $\mathrm{n}$ & $\%$ \\
Favorable & 30 & $40,6 \%$ & 20 & $27,0 \%$ & 50 & $67,6 \%$ \\
Muy favorable & 14 & $18,9 \%$ & 10 & $13,5 \%$ & 24 & $32,4 \%$ \\
Total & 44 & $59,5 \%$ & 30 & $40,5 \%$ & 74 & $100,0 \%$ \\
Prueba exacta de Fisher $=0,024$ & P-Valor $=0,000$ & & &
\end{tabular}

\section{Discusión}

Según las características maternas de las gestantes de la Micro Red Metropolitana de la Red de Salud Tacna, la mayoría de las gestantes tenía por ocupación ser amas de casa y sus edades oscilaban entre 18 a 35 años en mayor porcentaje, con respecto al estado civil prevalecen las gestantes en convivencia. Según el estudio de Guevara (9), en cuanto a las características de las participantes, los resultados muestran que el 7,7 \% era ama de casa y el $73,1 \%$ se encontraba entre $26-35$ años de edad. Esto en comparación a un estudio realizado en Nigeria por EO Ugwu et al. (10), cuyo objetivo fue identificar la tasa de cumplimiento y los determinantes de cumplimiento a la suplementación de hierro en gestantes. Los autores concluyeron que el grado de conocimientos estaba asociado significativamente a la ocupación y a la clase social.

Nuestro estudio también mostró que el 58,1\% de las gestantes presentaban anemia leve; esto se puede justificar a razón de que el nivel socio económico de las participantes era medio-alto. Por otro lado, al estudio de Barba-Oropeza y Cabanillas-Gurrola (11), tuvo como objetivo identificar los factores asociados a la anemia 
durante el embarazo. Los autores encontraron que uno de los factores asociados fue tener un nivel socio económico bajo. Esto también lo confirma un estudio realizado por Torres et al. (12), cuyo objetivo fue identificar los conocimientos, prácticas alimentarias y consumo de suplementos en gestantes de Antioquía Colombia, el cual evidenció que un bajo nivel educativo y bajos ingresos económicos se asoció con el incremento del riesgo de bajo consumo de: frutas y verduras en un $50 \%$, alimentos fuentes de hierro en un $40 \%$ y alimentos fuentes de calcio en un $20 \%$ y por ende mayor riesgo de tener anemia durante la gestación.

Con respecto al objetivo general, la presente investigación buscó determinar la relación entre el nivel de conocimientos y actitudes en alimentación saludable en gestantes con anemia. Los resultados obtenidos muestran que existe relación significativa entre la variable conocimientos sobre alimentación saludable y la anemia en las gestantes, según prueba exacta de Fisher $(\mathrm{p}<0,05)$. Estos resultados fueron similares con un estudio realizado por Karina E. Diez y Lucía S. Guerrero (13) en el 2010; el objetivo de ese estudio fue demostrar que el nivel de conocimientos, actitudes y prácticas sobre régimen dietario con hierro de la puérpera estaba relacionado a la presencia de anemia durante la gestación.

Guevara (9), respecto a la relación entre las variables conocimientos y la anemia en las gestantes entrevistadas, obtuvo resultados diferentes en su estudio. El autor mostró que no existe relación significativa entre el nivel de conocimientos sobre alimentación saludable y la anemia en las gestantes.

Por otro lado, Diez y Guerrero (13) encontraron que sí existe relación entre las actitudes sobre el régimen dietario con hierro y la anemia. Sin embargo, en el estudio de Guevara (9), respecto a la relación entre las variables actitud y la anemia en las gestantes entrevistadas, se mostró que no existe relación significativa entre el nivel de actitudes sobre alimentación saludable y la anemia en las gestantes.

Asimismo, se presenta el estudio de Diez y Guerrero (11), cuyo objetivo del estudio fue determinar los conocimientos, actitudes y prácticas, sobre régimen dietario con hierro y su relación con la anemia durante la gestación en puérperas atendidas en el Instituto Nacional Materno Perinatal (INMP). Los resultados mostraron que el conocimiento y las características del embarazo no mostraron relación significativa con la prevalencia de anemia a diferencia de las actitudes, las prácticas y el nivel económico, los cuales sí mostraron una relación significativa (11).

La muestra de estudio inicialmente se determinó en 86 gestantes con anemia, registrándose una pérdida de 12 casos durante el periodo de estudio debido a cambios de domicilio no determinado.

Como conclusión, se demuestra que existe relación significativa entre el nivel de conocimientos y actitudes sobre alimentación saludable y el nivel de anemia en las gestantes. 


\section{Referencias bibliográficas}

1. Peña, M. Gestión del conocimiento. Netbiblo. La Coruña. 2010.

2. Alavi, M. y Leidner, E. Knowledge Management and Knowledge Management Systems: Conceptual Foundations and Research Issues. MIS Quarterly, 2001;25 (1).

3. Organización Panamericana de la Salud (OPS). Manual de comunicación social para programas de comunicación de la salud de los adolescentes. 2001 . D is ponible [e n line a ] www.paho.org/spanish/ad/fch/adolpubs.htm Consultado [11 de junio del 2018]

4. Ortego, M., López, S., Y Álvarez, M. Las actitudes en ciencias psicológicas. Universidad de Cantabria. España; 1988.

5. Ministerio de Salud. Lineamiento de nutrición materno infantil del Perú. CENAN. Lima. 2013.

6. Ministerio de Salud. Guía de práctica clínica para el diagnóstico y Tratamiento de la Anemia por Deficiencia de Hierro en Niñas, Niños y Adolescentes en Establecimientos de salud del primer nivel de atención. (s/f). Disponible [en $1 \quad \mathrm{i} \quad \mathrm{n} \quad \mathrm{e} \quad \mathrm{a}$ ] http://bvs.minsa.gob.pe/local/MINSA/3932.pdf Consultado [05 de agosto del 2018]

7. Ministerio de Salud. Directiva sanitaria para la prevención y control de la anemia por deficiencia de hierro en gestantes y puérperas. [Internet]. kipdf.com. [citado 21 de junio de 2018]. Disponible en: https://kipdf.com/directivasanitaria-para-la-prevencion-y-control-de-laa $\mathrm{n}$ e $\mathrm{m} \mathrm{i} \mathrm{a}-\mathrm{por}-\mathrm{d}$ e f i c i e n c i a de 5afea2d48ead0e606f8b4614.html

8. Saidman, N.; Raele, M.; Basile, M.; Barreto L.; Mackinonn M.; Poy, M.; Terraza, R; López, L. (2012). Conocimientos, intereses y creencias sobre alimentación y nutrición en gestantes. Diaeta. Buenos Aires. Argentina.; 30(139); 18-27, abr.-jun.2012. tab Articulo en Español| LILACS | ID: 1il-653234 Disponible [en linea] http://www.scielo.org.ar/pdf/diaeta/v30n139/v30 n139a04.pdf. Consultado [20 de abril del 2018]

9. Guevara E. Nivel de conocimientos y actitudes sobre alimentación saludable relacionados con la anemia en gestantes que asisten al programa de psicoprofilaxis Baby Hope de la Clínica Good Hope, Lima, 2014. Universidad la Unión. Lima Perú. 2015 Disponible [en linea] https://repositorio.upeu.edu.pe/bitstream/handle/ UPEU/196/Evelin_Tesis_bachiller_2015.pdf?seq uence $=1 \&$ isAllowed $=y-[07$ de diciembre del $2018]$

10. EO Ugwu, AO Olibe, SN Obi, AO Ugwu. Determinants of compliance to iron supplementation among pregnant women in Enugu, Southeastern Nigeria. 2013. Niger J ClinPract [en línea] 2014, 17(5): 608-12. http://www.njcponline.com/article.asp?issn=1119 3077 ; year $=2014 ;$ volume $=17$; issue $=5 ;$ spage $=608$; epage $=612$; aulast $=$ Ugquwww.revinut.udea.edu.c o/index.php/nutricio/article/viewFile/16488/1431 2

11. Barba-O., y Cabanillas J. Factores asociados a la anemia durante el embarazo en un grupo de gestantes mexicanas. Arc Med Fam, 2007; 9 (4): 170-175.

12. Torres E, Jiménez G, Calderón G, Fabra J., López G., Franco M., et al. Conocimientos y prácticas alimentarias en gestantes asistentes al programa de control prenatal, en municipios del departamento de Antioquia, Colombia. 2010. Perspect Nutr Humana [en línea] 2012; 14, 185 $\begin{array}{lllllllllllllll}1 & 9 & 8 & \text {. } & \mathrm{D} & \mathrm{i} & \mathrm{s} & \mathrm{p} & \mathrm{o} & \mathrm{n} & \mathrm{i} & \mathrm{b} & \mathrm{l} & \mathrm{e} & \text { : }\end{array}$ www.revinut.udea.edu.co/index.php/nutricion/art icle/viewFile/16488/14312

13. Diez, K. y Guerrero, L. Conocimientos, actitudes y prácticas en puérperas sobre el régimen dietario con hierro y su relación con la anemia en la gestación, Instituto Nacional Materno Perinatal 2010. Universidad Nacional Mayor de San Marcos. Lima - Perú.2011.

\section{Correspondencia}

edithgodoyg2@hotmail.com
Fecha de recepción: 10 de agosto de 2019

Fecha de aceptación: 30 de noviembre de 2018 